microstructures of monocytes were observed by TEM and SEM, and titanium was identified by energy dispersion type X-ray spectroscopy (EDX). Adherent monocytes were pre-cultured with Alexa 568 dextran (A-Dex) to visualise endosomes. Results TNS exposure induced apoptosis of PBMC in the 7 days culture, the dose dependency of which was similar to asbestos, although apoptosis was not induced at the early stage of day 2 unlike asbestos. The apoptosis was inhibited by Q$\mathrm{VD}-\mathrm{OPh}$ pan-caspase inhibitor. Isolated $\mathrm{CD}^{+}{ }^{+} \mathrm{T}$ cells as well as monocytes showed apoptosis caused by TNS exposure, whereas monocytes showed giant vacuole formation prior to apoptosis. TNS-like compounds in vacuoles were observed by TEM, and SEM images showed rough surface of the inner layer of vacuolar membrane, in which titanium was identified by EDX. Most of vacuoles showed co-localization with fluorescence of A-Dex.

Conclusion These results indicate that TNS have toxic effect to cause caspase-dependent apoptosis of immune cells. In particular, TNS showed characteristic toxicity for monocytes, in which engulfed TNS were thought to enter into the endosomal pathway, leading to vacuole formation followed by apoptosis. Those findings suggest hazardous risk of occupational exposure to TNS.

\section{BIOLOGICAL EFFECTS OF CLOTH CONTAINING SPECIFIC ORE POWDER IN PATIENTS WITH POLLEN ALLERGY}

${ }^{1} \mathrm{~S}$ Lee*, ${ }^{2} \mathrm{H}$ Okamoto, ${ }^{1} \mathrm{~S}$ Yamamoto, ${ }^{1} \mathrm{~T}$ Hatayama, ${ }^{1} \mathrm{H}$ Matsuzaki, ${ }^{1} \mathrm{~N}$ Kumagai-Takei, ${ }^{1} \mathrm{~K}$ Yoshitome, ${ }^{1} \mathrm{Y}$ Nishimura, ${ }^{3} \mathrm{~T}$ Sato, ${ }^{4} \mathrm{Y}$ Kirita, ${ }^{2} \mathrm{Y}$ Fujii, ${ }^{1} \mathrm{~T}$ Otsuki. ${ }^{1}$ Dept. Hygiene, Kawasaki medical school, Kurashiki, Japan; ${ }^{2}$ Cosmic Garden Co., Ltd., Okayama, Japan; ${ }^{3}$ Specified Non-profit Organisation MEDICAL TECHNO OKAYAMA, Okayama, Japan; ${ }^{4}$ Okayama University Organisation for Research Promotion and Collaboration, Okayama, Japan

\subsection{6/oemed-2018-ICOHabstracts. 166}

Introduction Pollen allergy is major problem in Japan. The custom-homebuilding company, Cosmic Garden Co. Ltd., located in Okayama City, Japan was established in 1997 and uses specific natural ore powder (SNOP) in wall materials a to improve allergic symptoms. In this study, we surveyed biological effects of short term stay in a room surrounded by SNOP cloth.

Methods To investigate the biological effects of SNOP, patients with a pollen allergy were recruited to stay in a room surrounded by cloth containing SNOP (CCSNOP), and their symptoms and various biological parameters were compared with those of individuals staying in a room surrounded by control non-woven cloth (NWC). Each stay lasted $60 \mathrm{~min}$. Before and immediately after the stay, a questionnaire regarding allergic symptoms, as well as POMS (Profile of Mood Status) and blood sampling, was performed. Post-stay minus prestay values were calculated and compared between CCSNOP and NWC groups.

Results Results indicated that some symptoms, such as nasal obstruction and lacrimation, improved, and POMS evaluation showed that patients were calmer following a stay in CCSNOP. Relative eosinophils, non-specific Ig E, epidermal growth factor, monocyte chemotactic protein-1, and tumour necrosis factor- $\alpha$ increased following a stay in CCSNOP.

Conclusion This ore powder improved allergic symptoms, and long-term monitoring involving 1 to 2 months may be necessary to fully explore the biological and physical effects of SNOP on allergic patients.

\section{CLINICAL EVALUATION OF CENP-B AND SCL-70 AUTOANTIBODIES IN SILICOSIS PATIENTS}

${ }^{1} \mathrm{~S}$ Lee ${ }^{*},{ }^{2} \mathrm{H}$ Hayashi, ${ }^{1} \mathrm{~N}$ Kumagai-Takei, ${ }^{1} \mathrm{H}$ Matsuzaki, ${ }^{1} \mathrm{~K}$ Yoshitome, ${ }^{1} \mathrm{Y}$ Nishimura, ${ }^{3} \mathrm{~K}$ Uragami, ${ }^{4} \mathrm{M}$ Kusaka, ${ }^{1} \mathrm{~S}$ Yamamoto, ${ }^{1} \mathrm{M}$ Ikeda, ${ }^{1} \mathrm{~T}$ Hatayama, ${ }^{2} \mathrm{~W}$ Fujimoto, ${ }^{1} \mathrm{~T}$ Otsuki. ${ }^{1}$ Dept. Hygiene, Kawasaki medical school, Kurashiki, Japan; ${ }^{2}$ Dept. Dermatology, Kawasaki medical school, Kurashiki, Japan; ${ }^{3}$ Hinase Uragami lin, Bizen, Okayama, Japan; ${ }^{4}$ Kusaka Hospital, Okayama, Japan

\subsection{6/oemed-2018-ICOHabstracts. 167}

Introduction Silicosis patients (SIL) suffer from respiratory disorders and dysregulation of autoimmunity. Frequent complications such as rheumatoid arthritis, systemic sclerosis (SSc) and vasculitis are known in SIL. Furthermore, we reported previously that some SIL exhibited better respiratory conditions in association with a worse immunological status. In this study, the clinical roles of anti-CENP-B and Scl-70 autoantibodies in SIL were analysed.

Methods Plasma samples were collected from Healthy Volunteers (HV), SIL, Systemic Sclerosis (SSc). Plasma factors and autoantibodies were determined by ELISA. Statistical analysis were performed by SPSS (IBM).

Results The titer index ( $\log 10)$ of anti-CENP-B autoantibody in SIL was higher than that of HV and that of SSc was higher than those of HV and SIL. This titer index was positively correlated with an assumed immune status of 1 for $\mathrm{HV}, 2$ for SIL, and 3 for SSc. Moreover, although factor analysis revealed that the titer index of the anti-CENP-B autoantibody formed the same factor with the anti-Scl-70 autoantibody, IgG value and age in SIL cases, another extracted factor indicated that the $\operatorname{IgA}$ value and anti-Scl-70 antibody were positively related, but anti-CENP-B showed an opposite pattern in the results of the factor analysis.

Conclusion These findings indicated that the titer index of anti-CENP-B autoantibody may be a biomarker for dysregulation in SIL cases. Future clinical follow-up of SIL may therefore require both respiratory and immunological assessment

\section{Cardiology in Occupational Health}

\section{THE ASSESSMENT OF OCCUPATIONAL RISKS OF ACCELERATED AGEING AMONG RUSSIAN PROFESSIONAL DRIVERS}

1,2Bashkireva Angelika*. 'Gerontological Centre of Leningrad Region, St. Petersburg, Russia; ${ }^{2}$ Research and Innovation Centre "Professional Longevity», St. Petersburg, Russia

\subsection{6/oemed-2018-ICOHabstracts. 168}

Introduction We know the phenomenon of differential ageing to be the result of unequal environment conditions pressure. It is necessary to assess the difference between calendar and biological age (BA) in order to reveal the effects induced by combined occupational hazards in professional lorry-drivers. We have compared the physical (PWC) and mental work capacity (MWC), BA and ageing rates of lorry-drivers (experimental group) and labourers (control group) in connexion with their chronological age (CA), driving experience, occupational environment, work schedule and social-demographic characteristics.

Methods 150 male lorry-drivers (mean age $41.3 \pm 0.9$ ) and 150 male labourers (mean age 44.8 \pm 0.9 ) were examined according to the multiply-regressional model of BA evaluation based on 